\title{
Oral Hemorrhage, CTCAE
}

National Cancer Institute

\section{Source}

National Cancer Institute. Oral Hemorrhage, CTCAE. NCI Thesaurus. Code C56551.

A disorder characterized by bleeding from the mouth. 